\title{
Awareness of antenatal care clients on Mother-to-child- transmission (MTCT) of HIV infection and its prevention in Arba Minch
}

\author{
Mesfin Haddis ${ }^{1}$, Degu Jerene ${ }^{2}$
}

\begin{abstract}
This study was done on pregnant mothers attending the antenatal care in Arba Minch Hospital and Arba Minch Health Centre from June15-Aug 30, 2003. Four hundred and eighty four mothers were interviewed at the antenatal clinics. The majority of the mothers $(97 \%)$ were married. The risk of transmission of HIV, perinatally and through breastfeeding is well known to most mothers. However, the use of ARV prophylaxis was not known to 386(80\%) of the mothers. During interview 444(92.3\%) of the mothers did not know their HIV serostatus and, $360(74.4 \%)$ of all the mothers interviewed volunteered for HIV test. The results of this study indicate the need to integrate voluntary counselling and testing (VCT) in antenatal programmes and the need of educating mothers on the role of ARV prophylaxis in PMTCT. [Ethiop.J.Health Dev. 2006;20(1):55-57]
\end{abstract}

\section{Introduction}

Most sub-Saharan countries have been suffering with war, poverty, famine, and communicable diseases. It is rather unfortunate that while the problems still exist, the HIV/AIDS pandemic is causing more disasters than the others added together. The disease has created an enormous challenge on the survival of mankind. Since its recognition, the virus has infected close to 65 million individuals and over 25 million have already died due to AIDS (1). More than $70 \%$ of the over 42 million people living with the virus worldwide live in sub-Saharan Africa. AIDS has now become the leading cause of death in these countries (2) and it has further worsened the morbidity and mortality of infants and children, pertaining to its mother-to-child transmission (MTCT) risk. MTCT, which occur during pregnancy, labour, and breastfeeding, is responsible for $90 \%$ childhood HIV infection (3). Current estimate suggests that 600,000 children are newly infected annually worldwide and over 200,000 children below age 5 years are living with the virus in Ethiopia (4). The main objective of the prevention of mother-to-child transmission (PMTCT) is to reduce the transmission of HIV infection from HIV infected mothers to their off springs. However, it is to be noted that the most important public health measures against MTCT remain to be the prevention of infections in women of childbearing age and the prevention of unwanted pregnancies through adequate family planning. In a study done in North-western Ethiopia, although the general awareness of HIV was high, correct knowledge of the virus and its mode of transmission was known in only $44 \%$ of adolescent boys and $41 \%$ of adolescent girls (5). A sentinel surveillance reports of serostatus of pregnant women from five towns of southern Ethiopia revealed a prevalence of $7.8 \%$ (6). An increasing incidence of HIV in pregnant mothers would ultimately lead to increased incidence of HIV in children. The reasons for an increasing MTCT of HIV might include lack of knowledge of mothers on the risk of MTCT, lack of access to voluntary counselling and testing (VCT) and the benefits of preventive interventions, like antiretroviral (ARV) drugs and infant feeding options. ARV prophylaxis and avoiding breastfeeding are cornerstones of the strategy of PMTCT. In the absence of breastfeeding, most infections occur during labour, and delivery (7). MTCT in cohorts of women who have not received any preventive treatment ranges from $15 \%-25 \%$ in industrialized countries to $25 \%-45 \%$ in developing countries (8). Remarkable reduction in paediatrics HIV infection have been observed in industrialized countries since 1994, when it was proved that administration of Zidovudine to women in last trimester of pregnancy and during labour, and to the newborn, decreased the risk of MTCT by nearly $70 \%$ in the absence of breast feeding (9). This study aims to assess the awareness of pregnant mothers attending antenatal care (ANC) services of Arba Minch Hospital and Arba Minch Health Centre on MTCT of HIV infection, VCT and use of ARV drugs for PMTCT, in an effort to organize prevention of MTCT projects in the health institutions. The planned PMTCT activity would include the integration of VCT in antenatal programmes and initiation of ARV drugs to sero positive mothers. Hence, the knowledge of the mothers on different modes of MTCT, their HIV serostatus, and consent for counselling and testing (CT) services are studied along with their awareness on the role of ARV drugs in PMTCT. Intervention on infant feeding options would need to be studied carefully, considering affordability.

\section{Methods}

Antenatal care services are very strategic for preventive programmes of MTCT of HIV infections. For this reason our study has focused on pregnant mothers attending ANC of Arba Minch Hospital and Arba Minch Health Center, from June 15-Aug. 30, 2003. The study instru-

P.O. Box 40983, Tel. 493109, E-mail haddismesfin@yahoo.com, Addis Ababa, Ethiopia; ${ }^{2}$ Arbaminch Hospital, P.O. Box 28, Tel 06811691, E-mail ddao81@yahoo.com 
ment has been interviewing the pregnant mothers on their awareness of MTCT of HIV infections, its mode of transmission and prevention. There were questionnaires structured to every attendee, identifying the mothers by their clinical record number, age, marital status, occupation and address of residence. The MCH nurses of the antenatal clinics participated as interviewers after a brief orientation. There has been a pre- test to check the clarity and understand ability of the questionnaires and both groups were actively monitored during the pre-test. The mothers were requested to voluntarily participate in the interview and none of them refused to be interviewed. There was no exclusion. The interview was conducted before the start of the routine antenatal clinics and each mother was interviewed privately and assured on the confidentiality of the interview. The antenatal follow-up card of the interviewed mothers was marked to avoid repetition, and there were none repeated or missed cases in the study period. The filled formats were collected every day and the results analysed after completion of the study according to different variables in the format. The interview included the assessment of the knowledge of the mothers on transmission of HIV infection perinatally, through breastfeeding and physical contact. They were also inquired on their knowledge of the role of ARV drugs in PMTCT, and as well encouraged to voluntarily communicate if they have been tested for HIV infection and their attitude towards voluntary testing was described.

\section{Results}

Four hundred and eighty four mothers were interviewed at the antenatal follow-up of Arba Minch Hospital and Arba Minch Health Centre, from June15-Aug 30, 2003. The majority of the mothers (97\%) were married, from Arbaminch town (93.2\%), aged between 20-30 yrs $(61.2 \%)$ and housewives $(61.8 \%)$.

Eighty percent of all mothers are aware of the perinatal transmission of HIV infection and of these 213(55\%) thought that there is a $100 \%$ risk of transmission of every conception. Out of all the mothers interviewed, 424(91\%) agreed that HIV is not transmitted by mother-to-child physical contact, and $419(86.5 \%)$ have the knowledge that HIV is transmitted through breastfeeding (Table1).

It is to be noted that the use of ARV drugs in PMTCT was not known to $386(80 \%)$ of the mothers.

Even though 444(92.3\%) of the mothers did not know their HIV serostatus, 360(74.4\%) of all the pregnant mothers volunteered for HIV blood test at the first request (Table 1).

\section{Discussion}

ARV prophylaxis and infant feeding options remain to be key strategies in PMTCT. As interventions become more
Table 1: The knowledge of pregnant mothers on MTCT of HIV at the ANC of Arba Minch Hospital.

\begin{tabular}{lrr}
\hline & Number & Percent \\
\hline Aware about perinatal & & \\
transmission of HIV & 387 & 80.0 \\
$\quad$ Yes & 37 & 7.6 \\
No & 60 & 12.4 \\
$\quad$ Do not know & 484 & \\
$\quad$ Total & & \\
Aware about HIV transmission & & \\
through breastfeeding & 419 & 86.6 \\
Yes & 31 & 6.4 \\
No & 34 & 7.0 \\
Do not know & 484 & \\
Total & &
\end{tabular}

Aware about HIV transmission from mother to child by physical contact

$\begin{array}{lrr}\text { Yes } & 40 & 8.3 \\ \text { No } & 424 & 87.6 \\ \text { Do not know } & 20 & 4.1\end{array}$

Total

484

Aware about ARV drugs in mother to child transmission of HIV

Mother's HIV status Known

Yes

No

Total

Mother's HIV status known Yes

No

Total

484

Mother willing for HIV test

Yes

No

360

Not sure

Total

20.2

79.8

91.1

74.4

7.4

18.2

widespread, HIV infected women would have to know their HIV status in order to benefit from them. Therefore, counselling and testing $(\mathrm{CT})$ services would be required to be expanded in general and integrated in antenatal programmes in particular. In this study, less than $10 \%$ of the mothers knew their HIV status, however, the majority of the interviewed mothers volunteered to be tested. So far 76 mothers are tested upon their own request; however an integration of counselling and testing services is yet to be organized in the antenatal services. The lack of CT in ANC is a missed opportunity for PMTCT intervention and calls for integration of CT in antenatal programmes. In this study the risk of transmission of HIV, perinatally and through breastfeeding is well known to most mothers. However, the use of ARV drugs in PMTCT was not known to most of them. Literature is generally lacking on this topic, however, one study conducted in the general population in and around Addis Ababa, Ethiopia indicated that most of the respondents in the general population $(93.2 \%)$ 
were aware of the reality of HIV/AIDS but some (10.2\%) have never heard about how to prolong the life of such patients (10). Thus, much education is needed on the role of ARV prophylaxis in PMTCT. It would also expectedly encourage more mothers for VCT .ARV drugs have proved to be effective in PMTCT by reducing maternal viral load. In the USA, where ARV drugs are widely used to reduce perinatal transmission, the incidence of AIDS in infants, a sensitive indicator of MTCT, has fallen by $80 \%$ (11). In breast-feeding population, up to $20 \%$ of infants born to HIV infected mothers may acquire HIV through breastfeeding (7). However, due to the economic unaffordability and importance of breastfeeding to infant health in developing countries, cessation of breastfeeding must be considered only with the provision of alternatives to breastfeeding. In conclusion, the results of this study has emphasized on the importance of integration of counselling and testing in ANC and the need of educating mothers on the role of ARV prophylaxis in PMTCT. Further interventional services and studies are recommended based on the findings.

\section{Acknowledgement}

We are very much grateful to $\mathrm{MCH}$ nurse of the Arbaminch hospital, Sr. Fikrte Mekonnen, and the $\mathrm{MCH}$ staff of the health centre. We also extend our thanks to Professor Bernt Lindtjorn and the Norwegian Lutheran Mission (NLM) for organizing the HIV Project at Arba Minch Hospital and to Mrs. Mestewat who typed the manuscript.

\section{References}

1. Connor EM, Sperling S, Gelber et. al. Reduction of maternal-infant transmission of human immuno- deficiency virus type1 with Zidovudine treatment. N. Eng. J. Med 1994;331:1174-79.

2. Disease Prevention and Control Department, $\mathrm{MOH}$. AIDS in Ethiopia. Fourth edition, October 2002.

3. Msellanti P, Newell ML, Davis F.Rates of motherto-child transmission of HIV-1 in. Africa, America, and Europe. Results of perinatal studies. J Acquir Immune Defic Syndr 1995;8:506-10.

4. UNAIDS. Report on the global HIV/AIDS epidemic, Geneva: UNAIDS, 1998.

5. Alene GD, Wheeler JG, Grosskurth H. Adolesent reproductive health and awareness of HIV among rural high school students, North Western Ethiopia. AIDS care. 2004 Jan;16(1):57-68.

6. Disease prevention and control department, $\mathrm{MOH}$. AIDS in Ethiopia. Third edition, November 2000.

7. Decock KM, Fowler MG, Mercier E, et al. Prevention of mother-to-child HIV transmission in resource poor countries. Translating research into policy and practice. JAMA 2000; 283(9):1175-82.

8. UNAIDS. Report on the global HIV/AIDS epidemic, July 2002.

9. WHO ARV Treatment working group, scaling up antiretroviral therapy in resource limited settings. Guidelines for a public health approach, June 2002, WHO.

10. Yerdaw M, Nedi T, Enquoselassie F. Assessment of awareness of HIV/AIDS among selected target groups in and around Addis Ababa, Ethiopia. Afr $\mathrm{J}$ Reprod Health. 2002 Aug;6(2):30-8

11. Lindegren M, Byers RH, Thomas P, Davis SF et al. Trends in perinatal transmission of HIV/AIDS in the United States JAMA 1999;282:531-8. 
